\title{
Differential expression of IDA (INFLORESCENCE DEFICIENT IN ABSCISSION)- like genes in Nicotiana benthamiana during corolla abscission, stem growth and water stress
}

\author{
Daniel Ventimilla, Concha Domingo, Daniel González-Ibeas, Manuel Talon and Francisco R. Tadeo* (i)
}

\begin{abstract}
Background: IDA (INFLORESCENCE DEFICIENT IN ABSCISSION)-like signaling peptides and the associated HAE (HAESA)-like family of receptor kinases were originally reported in the model plant Arabidopsis thaliana (Arabidopsis) to be deeply involved in the regulation of abscission. Actually, IDA peptides, as cell-to-cell communication elements, appear to be implicated in many developmental processes that rely on cell separation events, and even in the responses to abiotic stresses. However, the knowledge related to the molecular machinery regulating abscission in economically important crops is scarce. In this work, we determined the conservation and phylogeny of the IDA-like and HAE-like gene families in relevant species of the Solanaceae family and analyzed the expression of these genes in the allopolyploid Nicotiana benthamiana, in order to identify members involved in abscission, stem growth and in the response to drought conditions.
\end{abstract}

Results: The phylogenetic relationships among the IDA-like members of the Solanaceae studied, grouped the two pairs of NbenIDA1 and NbenIDA2 protein homeologs with the Arabidopsis prepropeptides related to abscission. Analysis of promoter regions searching for regulatory elements showed that these two pairs of homeologs contained both hormonal and drought response elements, although NbenIDA2A lacked the hormonal regulatory elements. Expression analyses showed that the pair of NbenIDA1 homeologs were upregulated during corolla abscission. NbenIDA1 and NbenIDA2 pairs showed tissue differential expression under water stress conditions, since NbenIDA1 homeologs were highly expressed in stressed leaves while NbenIDA2 homeologs, especially NbenIDA2B, were highly expressed in stressed roots. In non-stressed active growing plants, nodes and internodes were the tissues with the highest expression levels of all members of the IDA-like family and their putative HAE-like receptors.

Conclusion: Our results suggest that the pair of NbenIDA1 homeologs are involved in the natural process of corolla abscission while both pairs of NbenIDA1 and NbenIDA2 homeologs are implicated in the response to water stress. The data also suggest that IDA peptides may be important during stem growth and development. These results provide additional evidence that the functional module formed by IDA peptides and its receptor kinases, as defined in Arabidopsis, may also be conserved in Solanaceae.

Keywords: Abiotic stress, Cell separation, Cis-acting regulatory elements, Gene expression, LRR-RLKs, Phylogeny, Signaling peptide, Solanaceae, Vegetative growth

\footnotetext{
* Correspondence: tadeo_fra@gva.es

Centro de Genómica, Instituto Valenciano de Investigaciones Agrarias (IVIA),

CV-315, Km 10,7 Moncada, E-46113 Valencia, Spain
}

(c) The Author(s). 2020 Open Access This article is distributed under the terms of the Creative Commons Attribution 4.0 International License (http://creativecommons.org/licenses/by/4.0/), which permits unrestricted use, distribution, and reproduction in any medium, provided you give appropriate credit to the original author(s) and the source, provide a link to the Creative Commons license, and indicate if changes were made. The Creative Commons Public Domain Dedication waiver (http://creativecommons.org/publicdomain/zero/1.0/) applies to the data made available in this article, unless otherwise stated. 


\section{Background}

The significance of the INFLORESCE DEFICIENT IN ABSCISSION (IDA)-like gene family is primary associated with the observation that AtIDA was deeply involved in the regulation of the abscission of floral organs and cauline leaves in Arabidopsis thaliana (Arabidopsis) [1-3]. Abscission is an active, organized and highly coordinated cell separation process allowing the detachment of entire vegetative and reproductive organs through the modification of cell-to-cell adhesion and breakdown of cell walls at specific sites on the plant body known as abscission zones (AZs), a discrete group of functionally specialized cells (for a review, see [4]). From an evolutionary point of view, abscission is a very favorable process that has several advantages such as seed dispersal as well as the shedding of no longer needed, damaged or infected organs. In addition, the abscission process is related to other processes such as senescence, pathogen defense and drought stress tolerance [5]. Abscission of aerial organs, on the other hand, may become a major limiting factor of yield in an agricultural context. It is widely accepted that the control of abscission in Arabidopsis requires physical interaction of the hormonal peptide AtIDA, a pair of redundant receptor-like protein kinases, HAESA (HAE) and HAESA- LIKE2 (HSL2), and SOMATIC EMBRYOGENESIS RECEPTOR-LIKE KINASE (SERK) co-receptors (for a review, see [6]). This interaction activates a signal transduction through a downstream MAP kinase cascade that leads to the expression of multiple abscission-related hydrolytic enzymes such as pectin-methylesterases, polygalacturonases, cellulases, xyloglucan-endotransglycosylase/hydrolases and expansins $[7,8]$. The release of this set of enzymes cause the disassembly of the cell wall and the dissolution of the middle lamella, resulting in the detachment of floral organs in Arabidopsis. Furthermore, in Arabidopsis ida mutants, petals remain indefinitely attached to the flower [1]. It has been also shown that synthetic IDA peptides were able to induce early floral abscission in Arabidopsis flowers [9].

The function of IDA peptides, as cell-to-cell communication elements, does not appear to be solely restricted to their roles in abscission. The activity of these peptides has also recently been involved in other developmental processes in Arabidopsis all of them settled on cell separation events such as the emergence of lateral roots and the root cap sloughing $[10,11]$. In addition, IDA peptides have recently been involved in the response to abiotic stresses. Expression analysis of Arabidopsis AtIDL6 and $A t I D L 7$, for instance, revealed that these two genes are rapidly induced during various stress treatments [12]. It was subsequently determined experimentally that these peptides were involved in the stress response as modulators of reactive oxygen species (ROS) signaling [13]. Furthermore, treatments with AtIDL6 and AtIDL7 peptides caused downregulation of important stressresponse key regulators like ZINC FINGER PROTEIN and WRKY transcription factors.

In addition to Arabidopsis, IDA-like genes have been identified in some crop species associated with organ abscission and the emergence of lateral roots [14] as well. Regarding organ abscission, particular members of the $I D A$-like gene family of tomato (SlIDA1), soybean ( $\mathrm{GmI}$ $D A 2 a)$, citrus (CitIDA3), litchi (LcIDL1), oil palm $(E g I D A 5)$ and yellow lupine (LlIDA) were highly expressed in leaf, flower or fruit abscission zones during abscission [15-19]. In addition, the AtIDA homologues of citrus (CitIDA3) and litchi (LcIDA1) were able to induce earlier floral organ abscission and to rescue the ida2 abscission deficiency when ectopically expressed in Arabidopsis $[16,20]$. All these data, together with the positive affect of treatments with synthetic IDA peptides on triggering organ abscission $[9,19,21]$ strongly suggest conserved functions of IDA-like genes in regulating cell separation events during organ abscission.

Solanaceae is a large plant family with approximately 90 genera comprising more than 3000 species found on almost all continents. Solanaceae is also one of the most economically important families worldwide. Some species of this family such as tomato (Solanum lycopersicum), potato (S. tuberosum), eggplant (aubergine; $S$. melongena) and pepper (Capsicum annum) are of great relevance as a human food source. Overall, more than 29 million hectares of these Solanaceae food species were cultivated globally in 2016, producing 644 million metric tons with a net production value of more than 146 billion US dollars (http://www.fao.org/faostat). Thus, in addition to being important in human nutrition, they are also relevant in economic and social terms. Other Solanaceae such as tobacco (Nicotiana spp.) have medical importance as a source of plant drugs while Nicotiana benthamiana is considered a relevant model organism for the study of plant-microbe interactions and also in plant molecular research and biotechnology [22, 23]. In this work, firstly, we determined the conservation and phylogeny of the IDA-like and HAE-like gene families by taking advantage of the free availability of the diploid genome sequences of tomato, eggplant, pepper, $N$. sylvestris, $N$. tomentosiformis, the allopolyploids $N$. tabacum and N. benthamiana, and the double haploid genome sequence of potato in the Solanaceae Genomic Network (SGN; https://solgenomics.net/). Allopolyploidy is a type of whole genome duplication derived from hybridization of two or more diverged taxa, that primarily occurs through the fusion of unreduced $(2 n)$ gametes. The result of this kind of genome merging is the occurrence of pairs of homologous genes from each of the diploid parents in the allopolyploid genome, called homeologs [24]. Therefore, in the allopolyploid genomes 
of $N$. tabacum and $N$. benthamiana we will likely find pairs of homeologs for many of the members of the gene families.

In order to identify and discriminate members involved in organ abscission, stem growth and in the response to drought conditions, we examined the expression of the homeolog genes of the $N$. benthamiana IDA-like and HAE-like families. The abscission of the corolla, the only organ that undergoes abscission in $N$. benthamiana, should be highly similar to that reported in N. tabacum [25]. The detachment of the corolla is due to the dissolution of the middle lamella and apparently to the disintegration of the parenchymal cells in its basal zone, a process that results in the detachment of the senescent corolla. Furthermore, the effect of water stress on the species of this contrasted genus has been the subject of major research and the physiological responses of these plants are also well known [26-29].

\section{Results}

\section{The IDA-like gene family in the Solanaceae}

Table 1 summarizes all $I D A$-like genes identified in our search in representative species of the genus Nicotiana such as $N$. sylvestris, $N$. tomentosiformis, $N$. tabacum and $N$. benthamiana in addition to other Solanaceae of agronomic interest such as tomato, potato, eggplant and pepper. All prepropeptides identified share two relevant characteristics, a signal peptide targeting the protein to the apoplast through the secretory pathway and a highly conserved C-terminal signature termed PIP motif typical of this gene family [1].

IDA-like families of the Nicotiana species $N$. sylvestris and $N$. tomentosiformis consisted of 5 members, while in the allopolyploids $N$. benthamiana and $N$. tabacum these families are formed by 5 pairs of homeologs, with one exception corresponding to NbenIDA4 whose homeolog pair was not found in the genomic screening. All IDA-like genes found in Nicotiana are new identifications, as the six members found in S. melongena and $C$. annuum and the seven members of the S. tuberosum family. In S. lycopersicum, five out of the eight IDA-like genes detected, members 1 to 5 , were already described in [15] and named SIIDA1-5, while the other three peptides, SlycIDA6-8, are incorporated in the current work.

\section{Phylogenetic relationship among IDA-like prepropeptides in Solanaceae}

The phylogenetic relationships among the IDA-like members of the species of Solanaceae studied, in addition to those of Arabidopsis, are grouped in three major clades (Fig. 1). Clade I (shadowed in green colors) was divided in two subclades. The subclade shadowed in green contained the two Arabidopsis prepropeptides involved in floral organ abscission, AtIDA and AtIDL1 [1,9]. The largest subclade grouped members of all eight Solanaceae species studied, as well as AtIDL8, the most divergent IDA-like peptide from Arabidopsis. In this subclade, Solanaceae members are further divided in two major groups. The group shadowed in lime green contained SIIDA1, the IDA-like member of tomato that has been associated with leaf abscission [15], other prepropeptides of potato (StubIDA4), eggplant (SmelIDA5) and pepper (CaIDA4) as well as the IDA1 members of the Nicotiana species under study. These were NsylIDA1 and NtomIDA1 of the diploid species $N$. sylvestris and $N$. tomentosiformis, respectively, and the two pairs of NtabIDA1 and NbenIDA1 homeolog prepropeptides corresponding to $N$. tabacum and $N$. benthamiana. The $5^{\prime}$-UTR regions and the predicted CDSs of all these IDA1 genes from the genus $\mathrm{Ni}$ cotiana showed high degree of conservation (see Additional files 1 and 3). The other group shadowed in light green included other prepropeptides from the Nicotiana, Solanum and Capsicum genera, with a small subdivision composed of AtIDL8 together with SlycIDA6, SlycIDA7 and StubIDA1 (Fig. 1). A second clade, clade II (shadowed in light orange), appeared to be limited to the Solanaceae family. This clade included prepropeptides from the Nicotiana, Solanum and Capsicum genera, but none from Arabidopsis, an observation suggesting that it might have diverged before the irruption of the Brassicaceae family 40 million years ago [33]. The third clade, clade III (shadowed in light gold), included AtIDL6 and AtIDL7, two IDA-like members of Arabidopsis that have been associated with processes different than cell separation, such as stress response [13]. The topology of the clade showed that there was a great diversification in Arabidopsis that generated at least six members, AtIDL2-7. It also included prepropeptides from the Nicotiana and Solanum genera, but none from Capsicum.

\section{Cis-acting regulatory elements in the promoter regions of the $N$. benthamiana IDA-like family}

Figure 2 shows a schematic representation of the cis-acting regulatory elements along $1000 \mathrm{bp}$ of the $5^{\prime}$-UTR region of the $I D A$-like family members of $N$. benthamiana and AtIDA and AtIDL1 of Arabidopsis. Searches for response elements to hormones related to ABA, methyl jasmonate (MeJa), AUXs or GAs, as well as response elements to biotic and abiotic stresses were performed. Interestingly, the pair of NbenIDA1 homeologs contained similar promoter regions carrying response elements to ABA, MeJa and AUX as AtIDA in similar locations; these phytohormones have been involved in the abscission process in different ways $[1,34,35]$. The pairs of NbenIDA1 and NbenIDA2 homeologs also carry drought response elements in their promoter regions. On the other hand, NbenIDA2B, NbenIDA3A, Nbe$n I D A 4$, and the pair of NbenIDA5 homeologs are 
Table 1 IDA-like gene families in species of the Solanaceae family (genome localization from different Sol Genomics Network databases [30]). All prepropeptides are predicted to be localized in the secretory pathway according to TargetP [31] and SignalP-5.0 [32]

\begin{tabular}{|c|c|c|c|c|}
\hline $\begin{array}{l}\text { Gene } \\
\text { name }\end{array}$ & $\begin{array}{l}\text { Genome } \\
\text { localization }\end{array}$ & Prepropeptide length (aa) & Predicted signal peptide length (aa) & $\begin{array}{l}\text { PIP } \\
\text { domain }\end{array}$ \\
\hline NsyllDA1 & Nsyl_KD945166.1:74265..74582 forward & 105 & 39 & PIPPSAPSKRHN \\
\hline NsyllDA2 & Nsyl_KD978144.1:88678..88980 reverse & 100 & 32 & PIPPSAPSKRHN \\
\hline NsyllDA3 & Nsyl_KD951180.1:40337..40579 forward & 80 & 32 & PIPPSAPSKRHN \\
\hline NsyllDA4 & Nsyl_KD977536.1:13349..13576 forward & 75 & 22 & PIPPSAPSQRHN \\
\hline NsyllDA5 & Nsyl_KD962079.1:38313..38564 forward & 83 & 30 & PIPASGPSRKHN \\
\hline NtomIDA1 & Ntom_KB972926.1:26032..26325 forward & 97 & 37 & PIPPSAPSKRHN \\
\hline NtomIDA2 & Ntom_KB954314.1:53025..53614 forward & 96 & 32 & PIPPSAPSKRHN \\
\hline NtomIDA3 & Ntom_KB969023.1:33965..34204 forward & 79 & 31 & PIPPSAPSKRHN \\
\hline NtomIDA4 & Ntom_KB956501.1:19193..19405 reverse & 70 & 22 & PIPPSAPSQRHN \\
\hline NtomIDA5 & Ntom_KB958630.1:30910..31161 reverse & 83 & 30 & PIPASGPSRKHN \\
\hline NtablDA1A & Ntab-BX_AWOK-SS18147:707412..707735 reverse & 107 & 48 & PIPPSAPSKRHN \\
\hline NtablDA1B & Ntab-BX_AWOK-SS9960:271769..272062 forward & 97 & 37 & PIPPSAPSKRHN \\
\hline NtabIDA2A & Ntab-BX_AWOK-SS12153:24919..25221 reverse & 100 & 32 & PIPPSAPSKRHN \\
\hline NtabIDA2B & Ntab-BX_AWOK-SS20685:67080..68370 reverse & 96 & 32 & PIPPSAPSKRHN \\
\hline NtabIDA3A & Ntab-BX_AWOK-SS473:166199..166441 reverse & 80 & 32 & PIPPSAPSKRHN \\
\hline NtablDA3B & Ntab-BX_AWOK-SS2799:946688..946927 forward & 79 & 31 & PIPPSAPSKRHN \\
\hline NtabIDA4A & Ntab-BX_AWOK-SS18001:26098..26325 forward & 75 & 22 & PIPPSAPSQRHN \\
\hline NtablDA4B & Ntab-BX_AWOK-SS12176:491822..492033 reverse & 70 & 22 & PIPPSAPSQRHN \\
\hline NtabIDA5A & Ntab-BX_AWOK-SS18104:315608..315859 reverse & 83 & 30 & PIPASGPSRKHN \\
\hline NtablDA5B & Ntab-BX_AWOK-SS9524:125323..125574 forward & 83 & 30 & PIPASGPSRKHN \\
\hline NbenIDA1A & Niben101Scf00570:62104..62373 reverse & 90 & 36 & PIPPSAPSK----- \\
\hline NbenIDA1B & Niben101Scf01338:640730..641035 forward & 101 & 35 & PIPPSAPSKRHN \\
\hline NbenIDA2A & Niben101Scf23219:7370..7663 reverse & 97 & 32 & PIPPSAPSKRHN \\
\hline NbenIDA2B & Niben101Scf03368:114599..114892 reverse & 97 & 32 & PIPPSAPSKRHN \\
\hline NbenIDA3A & Niben101Scf18667:206436.206678 forward & 80 & 32 & PIPPSAPSKRHN \\
\hline NbenIDA3B & Niben101Scf01 180:267334..267576 reverse & 80 & 32 & PIPPSAPSKRHN \\
\hline NbenIDA4 & Niben101Scf19133:87532..87771 forward & 79 & 25 & PIPPSAPSQRHN \\
\hline NbenIDA5A & Niben101Scf03848:699324..699575 forward & 83 & 30 & PIPASGPSRKHN \\
\hline NbenIDA5B & Niben101Scf02135:404883..405122 reverse & 79 & 26 & PIPASGPSRKHN \\
\hline SlyclDA1 & SL3.0ch05:4200134..4200439 forward & 101 & 36 & PIPPSAPSKRHN \\
\hline SlycIDA2 & SL3.0ch06:38623220..38623453 forward & 77 & 30 & PIPPSAPSKRHN \\
\hline SlycIDA3 & SL3.0ch04:5799910..5800149 forward & 79 & 27 & PIPPSSPSKRHN \\
\hline SlyclDA4 & SL3.0ch07:58068277..58068558 reverse & 93 & 34 & PIPPSAPSKRCN \\
\hline SlycIDA5 & SL3.0ch05:1629558..1629893 forward & 111 & 29 & LIPPSGPSRRHN \\
\hline SlycIDA6 & SL3.0ch09:540104.540379 reverse & 91 & 26 & PIPPSAPSCRSS \\
\hline SlycIDA7 & SL3.0ch09:546577..546855 reverse & 92 & 27 & PLPPSAPSCRSS \\
\hline SlyclDA8 & SL3.0ch11:533813..534061 reverse & 82 & 28 & PIPASGPSRKHN \\
\hline StublDA1 & PGSC0003DMB000000071:252349..252627 reverse & 92 & 27 & PIPPSAPSCRSS \\
\hline StubIDA2 & PGSC0003DMB000000131:879373..879621 forward & 82 & 28 & PIPASGPSRKHN \\
\hline StubIDA3 & PGSC0003DMB000000243:905236..905523 forward & 95 & 29 & PVPPSGPSRRHN \\
\hline StubIDA4 & PGSC0003DMB000000410:16621..16935 reverse & 104 & 36 & PIPPSAPSKRHN \\
\hline StubIDA5 & PGSC0003DMB000000420:159814..160050 forward & 78 & 26 & PIPPSSPSKRHN \\
\hline
\end{tabular}


Table 1 IDA-like gene families in species of the Solanaceae family (genome localization from different Sol Genomics Network databases [30]). All prepropeptides are predicted to be localized in the secretory pathway according to TargetP [31] and SignalP-5.0 [32] (Continued)

\begin{tabular}{lllll}
\hline $\begin{array}{l}\text { Gene } \\
\text { name }\end{array}$ & $\begin{array}{l}\text { Genome } \\
\text { localization }\end{array}$ & Prepropeptide length (aa) & Predicted signal peptide length (aa) & $\begin{array}{l}\text { PIP } \\
\text { domain }\end{array}$ \\
\hline StubIDA6 & PGSC0003DMB000000461:377302..377535 forward & 77 & 30 & PIPPSAPSKRHN \\
StubIDA7 & PGSC0003DMB000000592:149451..149714 forward & 87 & 34 & PIPPSAPSERCN \\
SmellDA1 & Sme2.5_00993.1:18248..18481 forward & 77 & 30 & PIPPSAPSKRHN \\
SmellDA2 & Sme2.5_04429.1:34294..34539 forward & 81 & 28 & PIPPSAPSLRHN \\
SmellDA3 & Sme2.5_04724.1:40347..40592 forward & 81 & 27 & PIPASGPSRKHN \\
SmellDA4 & Sme2.5_06686.1:19811..20078 forward & 85 & 25 & PIPPSAPSDRCN \\
SmellDA5 & Sme2.5_08129.1:7336..7444 forward & 102 & 34 & PIPPSGPSKRHN \\
SmellDA6 & Sme2.5_09763.1:10983..11228 reverse & 81 & 26 & PVPPSAPSDRCN \\
CaIDA1 & PepperUCD10Xch04:178438292..178438525 forward & 77 & 27 & PIPPSAPSKRHN \\
CaIDA2 & PepperUCD10Xch06:176812434..176812673 reverse & 79 & 29 & PIPPSAPSKRHN \\
CaIDA3 & PepperUCD10Xch11:6480406..6480714 forward & 102 & 33 & PIPPSGPSKRHN \\
CaIDA4 & PepperUCD10Xch11:4624209..4624499 forward & 96 & 35 & PIPPSAPSKRHN \\
CaIDA5 & PepperUCD10Xch11:6480457..6480714 forward & 85 & 18 & PIPPSGPSKRHN \\
CaIDA6 & PepperUCD10Xch11:27920042..27920356 reverse & 104 & 24 & PIPPSEPSPRHN \\
\hline
\end{tabular}

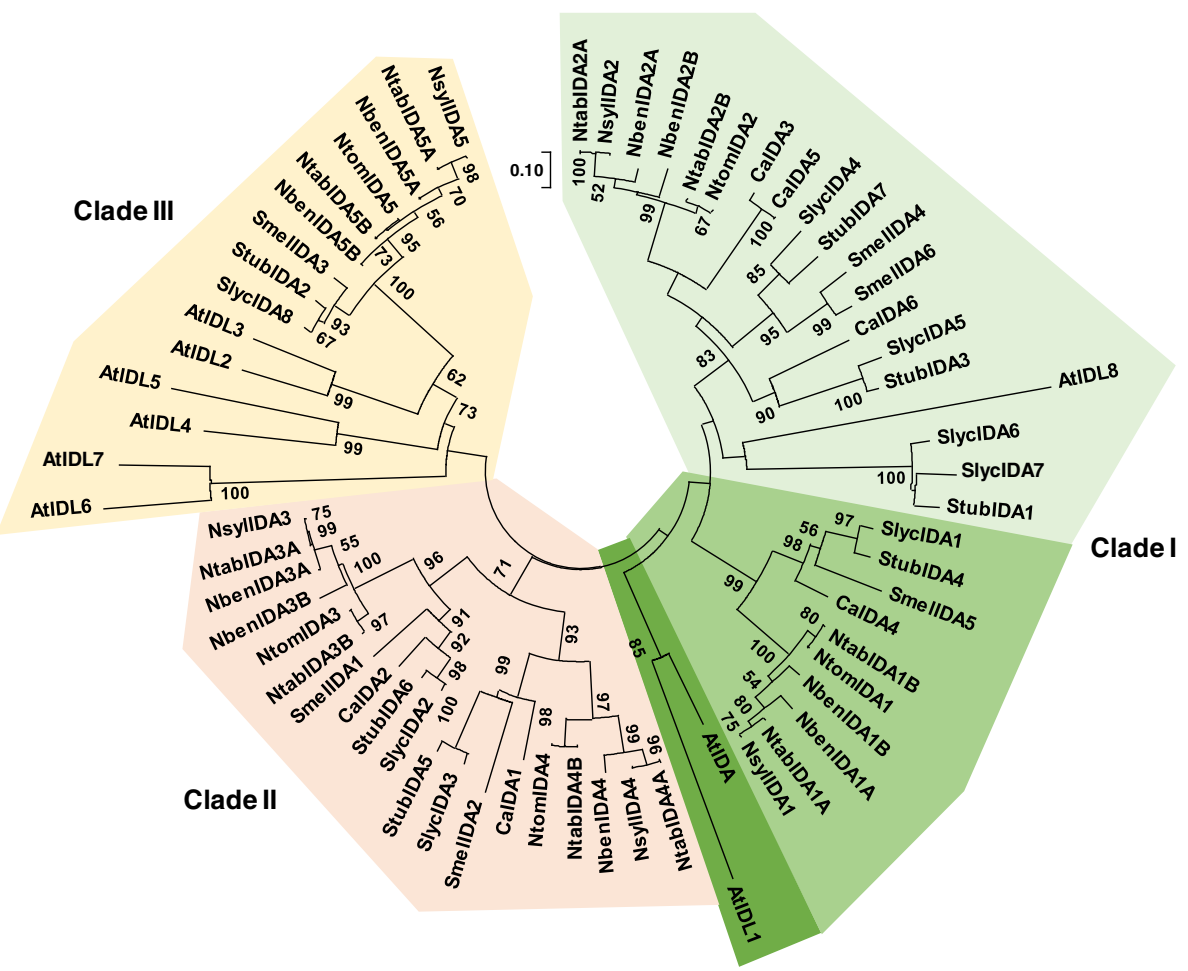

Fig. 1 Unrooted circular phylogenetic tree of IDA-like prepropeptides of Arabidopsis thaliana and relevant species of the Solanaceae family such as N. sylvestris, N. tomentosiformis, N. benthamiana, N. tabacum, S. Iycopersicum, S. melongena, C. annuum and S. tuberosum. Clade I shadowed in green color is divided in two subclades containing abscission-related genes (shadowed in lime green) and another one that grouped members of all eight Solanaceae species studied, as well as AtIDL8. The last subclade is subdivided in a group containing genes from Nicotiana, eggplant and pepper closed to SIIDA1, a tomato gene previously associated to abscission (shadowed in lime green) and other group (shadowed in light green) including from the Nicotiana, Solanum and Capsicum genera, with a small subdivision composed of AtIDL8 together with peptides from S. lycopersicum and S. melongena. Clade II (shadowed in light orange) includes exclusively Solanaceae genes. Clade III (shadowed in light gold) includes most of the Arabidopsis IDA-like family as well as Nicotiana and Solanum IDA-like genes. Bootstrap values are shown in each node 


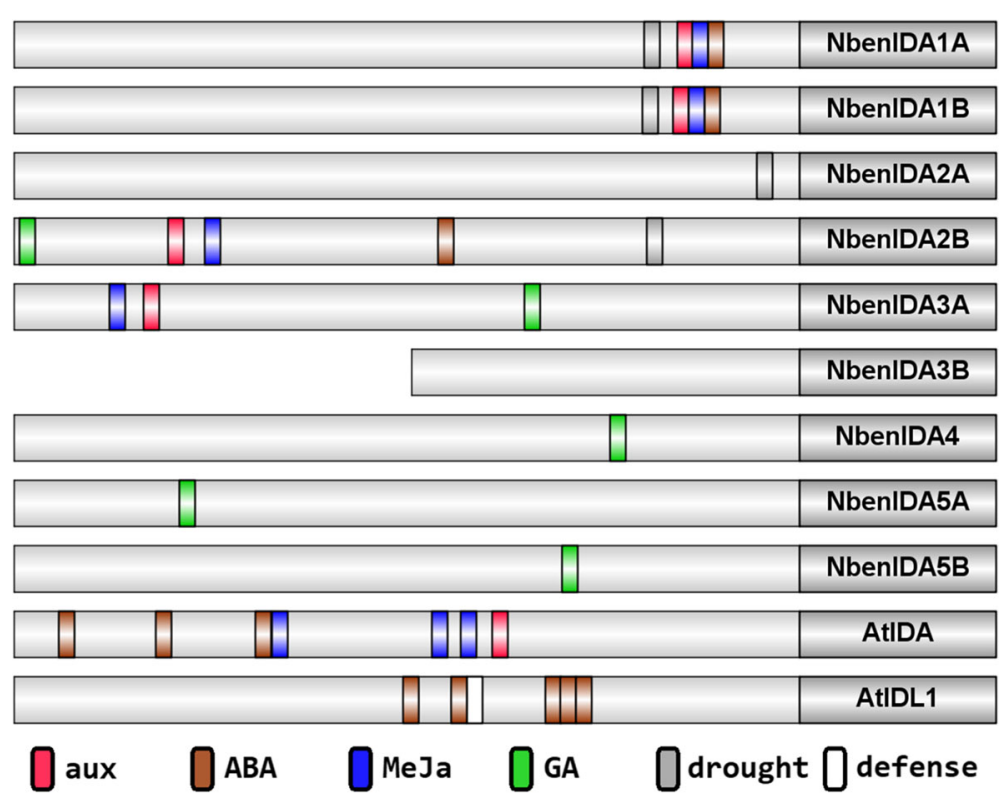

Fig. 2 Schematic representation of cis-acting regulatory elements of the promoter regions of the $\mathrm{N}$. benthamiana IDA-like gene family and Arabidopsis AtIDA and AtIDL1. Light grey boxes represent 1000 bp long promoter regions while dark grey boxes represent the 5' part of the gene. In-silico searches of response elements to hormones related to abscission such as abscisic acid, methyl jasmonate, auxins or gibberellins, as well as response elements to biotic and abiotic stresses were performed. Response elements color scheme: red, auxins; brown, abscisic acid; blue, methyl jasmonate; green, gibberelins; grey, drought stress; white, defense response. The promoter region of NbenIDA3B is $493 \mathrm{bp}$ long since the rest of the sequence is not available yet

characterized by the occurrence of GA response elements (Fig. 2).

\section{Expression patterns of IDA-like and HAE-like genes in Nicotiana benthamiana during growth and abscission} Expression analysis of the family of $I D A$-like ligand peptides and their putative $H A E$-like receptors in different plant tissues of $N$. benthamiana are presented in Fig. 3. HAE-like receptors were identified through the analyses of the phylogenetic relationships between the $H A E$-like receptor-like kinases (RLKs) of Arabidopsis and Nicotiana (see Additional files 2 and 3). The plant material selected for gene expression analysis is shown in the panel A of Fig. 3. This plant material included different vegetative tissues of a plant in active growth (apical buds, young and mature leaves, nodes and internodes, and roots), as well as reproductive tissues (anthers, styles, stigmas, and fruits) at different developmental stages, including samples of the base of the flower corollas, a tissue that in tobacco ( $N$. tabacum) has been shown to respond to the abscission process [25]. Panel B in Fig. 3 shows the expression of the N. benthamiana IDA-like and HAE-like homeologs in apical buds, nodes, internodes, the whole corolla, the ensemble formed by the stigma and the style and also in roots relative to the lowest expression level of each gene. Panel $\mathrm{C}$ in Fig. 3 shows the expression pattern of each $I D A$-like and $H A E$ like homeolog in leaves, anthers and fruits relative to that at the earliest developmental stage in every organ and panel D shows the expression patterns of each homeolog gene at the corolla base in developmental stages 2,4 and 5 relative to the corolla developmental stage 1 . Virtually all members of the IDA-like family of $N$. benthamiana were mainly expressed in nodes and internodes, although NbenIDA1A expression levels were not especially high in internodes (Fig. 3b). No changes in the expression patterns of $I D A$-like homeolog genes were observed in leaves and fruits (Fig. 3c) but the expression level for all of them except NbenIDA3B and NbenIDA4 showed a tendency to increase between closed and dehiscent anthers (Fig. 3c). Interestingly, expression of both NbenIDAI homeologs at the base of the flower corolla increased with the stage of development of the tissue, in parallel to the progress of the abscission process (Fig. $3 \mathrm{~d}$ ). The expression pattern of $N b e n I D A 1 B$ was similar to that detected in NbenIDA1A although at a much more modest level. The expression levels of the NbenIDA2 homeologs were transiently high in stage 2 when the corolla tube is fully elongated and the limb lobes are still closed to return later in stages 4 and 5 to almost the basal level of expression (Fig. 3d).

The highest expressions levels of the putative receptors of the IDA-like peptides, NbenHAE.1, NbenHAE.2, NbenHSL2.1 and NbenHSL2.2, were also registered in nodes and internodes (Fig. 3b). Additionally, their expression levels also showed a tendency to increase between closed and dehiscent anthers (Fig. 3c) and a slight increase was observed at the corolla base associated with corolla development (Fig. 3d). 


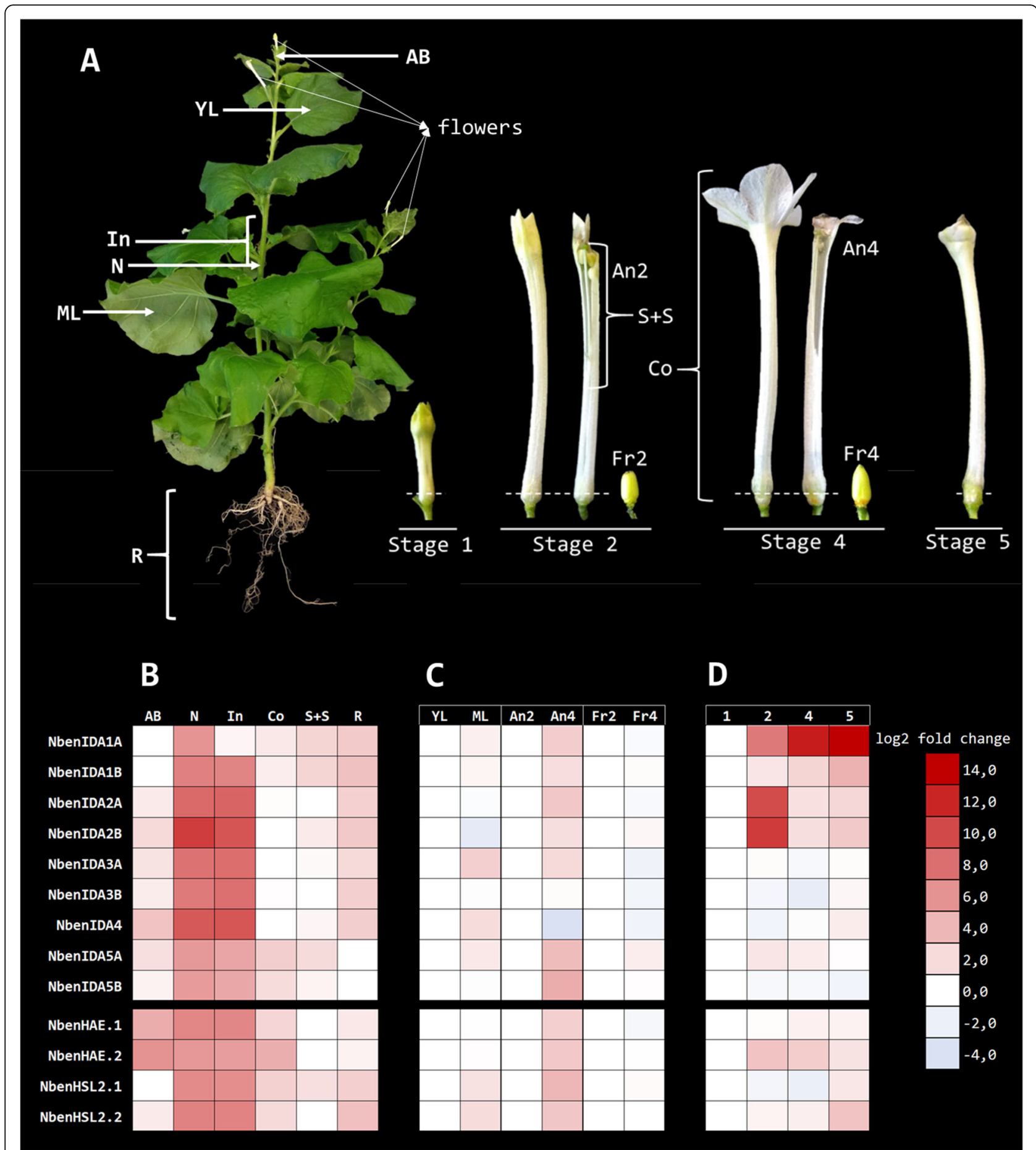

Fig. 3 (See legend on next page.)

Expression patterns of IDA-like genes in Nicotiana benthamiana during water stress

The presence of drought response elements in the promoter regions of some particular $I D A$-like members, e.g. NbenIDA1A, NbenIDA1B, NbenIDA2A and NbenIDA2B (Fig. 2), suggested that their expression might be regulated by the water status of the plant. Therefore, we exposed actively growing plants of $N$. benthamiana to 6 (mild stress) and 8 (severe stress) days of water stress and the expression levels of all members of the $I D A$-like family in axillary buds, roots and leaves were determined (Fig. 4). While no differences in gene expression were 
(See figure on previous page.)

Fig. 3 Expression patterns of IDA-like and HAE-like genes based on quantitative real-time PCR in several organs and tissues of $N$. benthamiana at different stages of corolla development. a Floral organs (sepals were removed), fruits and vegetative tissues utilized for gene expression analysis. The developmental stages of the corolla that were selected as a source of floral organs and fruits showed the corolla tube bulb above the calyx and the corolla limb closed (stage 1); fully elongated corolla tube and closed corolla limb and anthers (stage 2); open flower, corolla limb fully expanded and anthers dehiscent (stage 4); lobes of the corolla limb curl inwards, significant loss of turgor of the corolla tube which easily detached from the receptacle (stage 5). Dash lines mark the tissue collected from the base of the corolla. $\mathbf{b}$ Gene expression levels in apical buds $(A B)$, nodes $(N)$, internodes $(I n)$, whole corolla $(C O)$, the ensemble formed by the style and the stigma $(S+S)$ and roots $(R)$ relative to the lowest expression level of each gene. c Gene expression patterns in leaves, anthers and fruits. Gene expression levels were relative to that at the earliest developmental stage in every organ (YL, young leaf; An2, anthers at stage 2; Fr2, fruit at stage 2, respectively). $\mathbf{d}$ Gene expression patterns in the corolla base during corolla developmental stages 1, 2, 4 and 5: Gene expression levels were relative to that at stage 1. Gene expression levels were normalized with respect to those of NbenPP2A gene, applying the $2^{-\Delta \Delta C t}$ method. Relative gene expression levels (log2 fold change) are given next to the color scale column. Upregulation and downregulation of gene expression is shown by red and blue color, respectively. Gene expression raw and processed final data are shown in Additional File 5

found in axillary buds, those of the pair of NbenIDA1 homeologs dramatically increased in leaf blades of plants subjected to severe water stress. In contrast, this condition resulted in higher increases in transcripts belonging of both NbenIDA2 homeologs in roots, indicating differential roles of this gene family in response to water stress. Changes in the expression of the rest of genes were of minor relevance although it is worth to mention that these members tended to repress their expression levels in roots of plants subjected to water stress, although NbenIDA5A expression was also reduced in stressed leaves.

\section{Discussion}

$I D A$-like genes were searched in relevant genera of the Solanaceae family including several species of Nicotiana (N. sylvestris, $N$. tomentosiformis, $N$. tabacum and $N$. benthamiana), and other crops of agronomic interest such as tomato (Solanum lycopersicum), potato (S. tuberosum), eggplant (S. melongena) and pepper (Capsicum annuum) (Table 1). This gene family was identified in a large number of Angiosperms [17] and their members contained a signal peptide targeting the protein to the apoplast through the secretory pathway and a conserved C-terminal part, the PIP motif (Additional file 4). The presence of a signal peptide in the sequence of all identified genes suggests a mechanism of posttranslational maturation in the apoplast similar to that described in Arabidopsis, where the prepropeptide is proteolytically processed by subtilisin-like serine proteinases to yield a bioactive peptide [36].

The reduced size of the mature IDA-like peptides (an alignment of the complete coding sequences of these genes can be seen in Additional file 4), precluded the study of the phylogenetic relationships based on these premises and, therefore, a circular phylogenetic tree was generated using complete sequences encoding prepropeptides including a signal peptide and a variable region (Fig. 1). This tree shows that the Arabidopsis IDA-like gene family exhibits a higher degree of diversification than the Solanaceae genera studied, with the exception of Nicotiana. The two Arabidopsis prepropeptides related to abscission, AtIDA and AtIDL1 [1, 34], nested in a small clade grouped with a larger clade including many IDA-like members from the different species of Solanaceae. This large clade was divided in two subgroups in one of which was nested the tomato SlycIDA1, associated with abscission [15], and its homolog genes in $S$. melongena, C. annuum, S. tuberosum and in the four $\mathrm{Ni}$ cotiana species studied. The genus Nicotiana originates in South America and its members have spread over four continents. This genus consists of diploid species and several allopolyploid species of different ages and paternity. Thus, cultivated tobacco ( $N$. tabacum) is an allotetraploid $(2 \mathrm{n}=4 \mathrm{x}=48)$ representing a hybridization event involving the diploid species $N$. sylvestris $(2 \mathrm{n}=$ $2 \mathrm{x}=24)$ and $N$. tomentosiformis $(2 \mathrm{n}=2 \mathrm{x}=24)$ as their female and male parentals, respectively. This hybridization event is believed to have occurred recently, less than 200,000 years ago [37, 38]. The fact that all homeologs of the IDA-like family of $N$. tabacum have corresponding counterparts in $N$. sylvestris and $N$. tomentosiformis (Table 1 and Fig. 1) is in line with this observation (Fig. 1).

In contrast to tobacco, $N$. benthamiana has been described as an ancient allotetraploid whose polyploidy level $(2 \mathrm{n}=4 \mathrm{x}=38)$ likely evolved through genome rearrangements and fractionation giving rise to a remarkable descending dysploidy [38]. Parentals of $N$. benthamiana are unknown, although it is believed that it comes from a hybridization event that occurred $>10$ Myr ago between species belonging to the Sylvestres and Noctiflorae sections of Nicotiana [38]. Two homeologs were identified for most of the analysed genes in our work, except for NbenIDA4. Genomic responses to polyploidy are complex in Nicotiana species, ranging from small to large genome re-sizing depending on the polyploid age and the similarity of parental genome donors [38]. Reduction in the number of chromosomes in $N$. benthamiana strongly suggests a considerable genome 


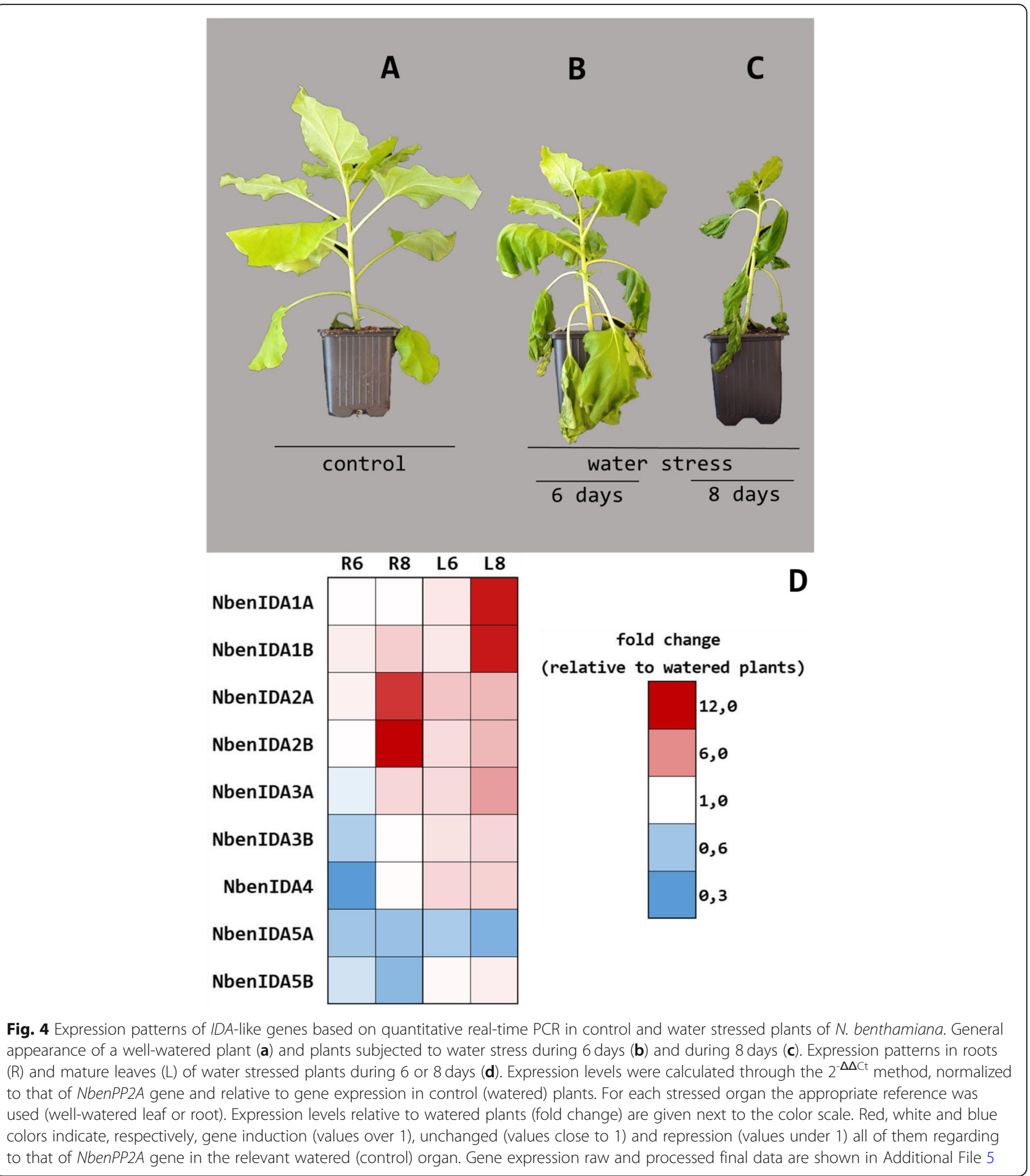

downsizing, probably as a consequence of being an older polyploid. The size reduction involves $1 \mathrm{~Gb}$ in length relative to tobacco $(4.5 \mathrm{~Gb}$ genome size of $N$. tabacum, $3.5 \mathrm{~Gb} N$. benthamiana). Therefore, we suggest that gene loss might explain the absence of a second copy of NbenIDA4 in our data, rather than a misrepresentation in the draft assembly of the genome used for the analysis. Interestingly, several genetic studies estimated that the genome of $N$. tabacum had lost DNA from its progenitors since polyploidization and that this genomic loss was greater and biased towards the genome of the male parental $N$. tomentosiformis [38-41]. Therefore, we also think that similar biased gene loss may have happened in $N$. benthamiana involving the copy of 
NbenIDA4 belonging to the parent of the Noctiflorae section of Nicotiana.

The analyses of the cis-acting regulatory elements in the $5^{\prime}$-UTR regions of the $N$. benthamiana IDA-like family and Arabidopsis AtIDA and AtIDL1 (Fig. 3) failed to identify ethylene response elements, in agreement with the idea that IDA-like genes regulating the abscission process are not directly dependent upon ethylene $[42,43]$. In contrast, the presence of response elements to AUXs, ABA, MeJa and GAs in the promoter regions of the IDA-like family members of $N$. benthamiana and AtIDA and AtIDL1 suggests that these phytohormones might play a role in regulating the expression of these genes. The occurrence of functional indole-3-acetic acid (IAA) signaling in the abscission zone during organ separation, for instance, has been demonstrated by Basu and co-workers [44]. It has also been determined that ABA and MeJa have abscission-promoting effects, while the role of GAs is not entirely clear $[6,45]$. However, it has been shown in citrus that flower pollination increased bioactive gibberellin A1 $\left(\mathrm{GA}_{1}\right)$ levels and reduced ovary abscission and that the treatment of unpollinated ovaries with $\mathrm{GA}_{3}$ also suppressed ovary abscission [46, 47].

Our bioinformatic analyses also indicated that the coding and promoter sequences of the pair of $\mathrm{Nbe-}$ nIDA1 homeologs are highly similar and that promoters share the same hormonal response elements in similar positions, in addition to the same drought response element (Fig. 2). High conservation of the coding and promoter sequences of IDAI duplicated genes in $N$. sylvestris, $N$. tomentosiformis and $N$. tabacum (see Additional file 3) suggests that they may be very important in the regulation of cell separation processes and response to stressful conditions. Furthermore, the pair of NbenIDA2 homeologs also contains drought stress response elements in their promoter regions (Fig. 2). Likewise, the coding and promoter sequences of the IDA1 genes in $N$. attenuata, $N$. sylvestris, $N$. tomentosiformis, $N$. benthamiana and $N$. tabacum are very similar and have the same response elements in the same positions in their promoters, except $N$. attenuata (Additional file 1).

The results described above (Fig. 1) suggested that in $N$. benthamiana, NbenIDA1A and NbenIDA1B peptides may be involved in the abscission process. This suggestion is also supported by the gene expression patterns found at the corolla base of the flowers during the process of natural abscission (Fig. 3d). Similarly, there seems to be a correlation between the expression of the $I D A$-like genes and that of their putative receptors of the HAE-like family, NbenHAE.1, NbenHAE.2 and NbenHSL.2.2 (Fig. 3d), that also increased during the last phases of the corolla abscission.
As described for IDA-like families in other species [12], the different members of the $N$. benthamiana family are also expressed in multiple plant tissues (Fig. 3). This is not a surprise since the IDA-like signaling peptides, as cell-tocell communication elements, function in several cell separation events, including lateral root emergence and root cap sloughing [10, 11]. Interestingly, in plants of $N$. benthamiana actively growing, the highest expression level of most members of the $I D A$-like family was found in nodes and internodes. It is worth mentioning that the promoter regions of NbenIDA2B, NbenIDA3A, NbenIDA4, NbenIDA5A and NbenIDA5B genes contain GAs response elements, and that these hormones are pivotal regulators of stem growth [48]. Moreover, all HAE-like genes analyzed also show higher expression levels in nodes and internodes, in parallel with the pattern observed for the IDA-like genes. These expression patterns might be linked to the formation of vascular bundles and to the cell elongation and division associated with the process of stem elongation implying cell wall remodeling.

The occurrence of cis-acting elements related to the drought response in the two pairs of NbenIDA1 and NbenIDA2 homeologs (Fig. 2) also suggested to test the response of the $I D A$-like genes to water stress conditions. In the experiment reported in Fig. 4 it is clearly observed that the first pair of homeologs was highly expressed in leaves from $N$. benthamiana plants severely stressed while in roots, the genes that responded to water deficit were the members of the second pair. Furthermore, these two pairs of homeologs are phylogenetically close to AtIDA and AtIDL1, two Arabidopsis genes that are induced under abiotic stress conditions [12].

Our gene expression data showed that while most pairs of homeologs showed similar expression patterns, some of them exhibited divergence in certain organs and tissues studied (see Fig. 3). This might well be linked to the frequent observation that some duplicated genes, after a whole genome duplication event, evolve to undertake different functions or partition the function of the ancestral gene in a process of subfunctionalization. This process can include epigenetic, coding sequence or promoter modifications that alters regulatory mechanisms (e.g. silencing) and give rise for example to differential level of expression or tissue specificity. Subfunctionalization becomes more relevant when gene dosage is not an adaptive advantage for the polyploid [49]. Therefore, our gene expression data might be revealing a putative subfunctionalization for the homeolog pairs NbenIDA1 at the corolla base and NbenIDA3 in leaves and anthers. We took special care in primer specificity during qPCR assays in order to distinguish between both homeologs, since gene expression artifacts may be recurrent among genes derived from genome duplicated areas due to high sequence similarity. 
It has been recently observed that IDA signaling peptides can certainly regulate important developmental processes as well as fundamental plant responses to environmental conditions [13]. Our data indicate that in the allopolyploid $N$. benthamiana, the two pairs of Nbe$n I D A 1$ and NbenIDA2 homeologs are differentially involved in the responses to drought stress while only NbenIDA1 homeologs are apparently implicated in the natural process of corolla abscission. These data suggest that IDA-like signaling peptides can play different biological roles in various tissues and under distinct abiotic conditions.

\section{Conclusions}

We have investigated the IDA-like and $H A E$-like gene families of different Solanaceae species, S. lycopersicum, S. melongena, C. annuum, S. tuberosum, and four species of the genus Nicotiana, N. sylvestris, N. tomentosiformis, $N$. benthamiana, and N. tabacum and determined their phylogenetic relationships. In the allopolyploid $N$. benthamiana, specific analyses of the the cis-acting regulatory sequences and the examinations of the gene expression patterns of the $I D A$-like family have identified putative candidate $I D A$-like genes implicated in corolla abscission and in the response to water stress. The results suggest that the pair of NbenIDA1 homeologs are both involved in the natural process of corolla abscission. Interestingly, they also show specific differential expression under water stress conditions. NbenIDA1 homeologs are highly expressed in stressed leaves while NbenIDA2 homeologs, especially NbenIDA2B, are highly expressed in stressed roots. In addition, nodes and internodes are the tissues with the highest expression of the $I D A$-like and $H A E$-like genes in normal active growing plants, suggesting that these peptides are also essential during stem growth and development. These results add new evidence that the functional module formed by IDA-like peptides and its receptor kinases as defined in Arabidopsis, may be conserved in Solanaceae.

\section{Methods}

\section{Retrieval and sequence analysis}

The EPIP motif of AtIDA (FGYLPKGVPIPPSAPSKRHNSFVNSLPH) was used to identify the $I D A$ like members of the selected Solanaceae species (N. sylvestris, $N$. tomentosiformis, $N$. tabacum, $N$. benthamiana, Solanum lycopersicum, S. tuberosum, S. melongena and Capsicum annuum) by tBLASTn and BLASTp inquiries in the Sol Genomics [50] web platform (https://solgenomics.net/tools/blast/), depending on the databases status. "N.sylvestris Genome", "N.tomentosiformis Genome", "N.tabacum BX Genome", "N.benthamiana v1.0.1", "Tomato ITAG release 3.20", "Potato PGSC DM v3 scaffolds", "Eggplant draft genome (release 2.5.1)" and
"Capsicum annuum UCD10X genome chromosomes (v1.0)" databases [30, 51-55] were used, respectively. Arabidopsis AtHAE, AtHSL1 and AtHSL2 protein sequences were retrieved from Phytozome v12.1, TAIR10 database and were used to identify the $H A E$-like members of the selected Solanaceae species in the same way as described above. Newly identified genes were named numerically, adding an "A", "B", ".1" or ".2" termination to the $I D A$-like or $H A E$-like gene pairs for the allopolyploids N. tabacum and N. benthamiana.

Sequence alignments were performed through MEGA7 software [56] using the ClustalW algorithm with default parameters (DNA Data Bank of Japan, DDBJ; http://clustalw.ddbj.nig.ac.jp/). Phylogenetic trees were created using the Neighbor-Joining method [57] using 1000 bootstrap replicates. The trees are drawn to scale, with branch lengths in the same units as those of the evolutionary distances used to infer the phylogenetic trees. The evolutionary distances were computed using the Poisson correction method [58] and are in the units of the number of amino acid substitutions per site. All ambiguous positions were removed for each sequence pair (pairwise deletion option).

Peptide localization prediction and presence of signal peptides in the IDL amino acid sequences were analyzed using the TargetP [31] and SignalP-5.0 [32] services. Up to 1000 base pairs of promoter regions upstream of the start codon of the IDL genes of the Nicotiana species available in Sol Genomics databases were retrieved and submitted for cis-acting regulatory element analysis in PlantCARE [59]. Schematic representations of regulatory elements of the promoter sequences where created using IBS1.0.3 software [60]. All DNA and protein sequences, as well as the localization of the sequences in the genomes in SolGenomics and the sequence IDs are contained in Additional file 7.

\section{Plant materials and growth conditions}

$N$. benthamiana seeds were obtained from Dr. José Guerri and Dr. Karelia Velázquez of the Centro de Protección Vegetal y Biotecnología (IVIA, Moncada, Spain). The seeds were germinated on nutrient soil and transplanted individually in small pots with an artificial potting mix (50\% vermiculite and $50 \%$ peat moss) in a plant growth chamber at $20 / 24{ }^{\circ} \mathrm{C}$ (night/day), $60 \%$ relative humidity and a 16/8-h light/dark regime. Water stress was induced by not watering the plants for 6 and 8 days for mild and severe stress conditions respectively.

\section{RNA extraction}

Basal portion of the corollas at different flower development stages as well as the rest of tissues used in gene expression analysis were manually collected from the plants and frozen with liquid nitrogen. Tissues were 
grinded using Thomas Scientific's Liquid Nitrogen Cooled Mortar. Total RNA was extracted using Macherey-Nagel's NucleoSpin ${ }^{\circ}$ RNA Plant, following the manufacturer's instructions. cDNA was synthesized from the RNA extraction using Thermo Fisher Scientific's SuperScript $^{\text {tm }}$ II Reverse Transcriptase, following the manufacturer's instructions.

\section{qPCR analysis}

Quantitative PCR analysis were performed using LightCycler ${ }^{\circ}$ FastStart DNA MasterPLUS SYBR Green I reaction mix and a LightCycler 2.0 instrument (Roche, Basel, Switzerland) using gene-specific primers designed based on transcriptome sequences using the Primer3Plus software [61]. Primer pairs are listed in Additional file 6 . Specificity of all the primer pairs used in this work was assessed by primer BLAST and melting curve analysis $[62,63]$. The fluorescence intensity data was obtained through LightCycler Software version 4.1. The $N$. benthamiana housekeeping gene NbenPP2A [64] was used for normalization in all qPCR reactions carried out in this work. Three biological replicates were run for assessing the expression values of each gene. The averaged expression values were obtained in the form of $\mathrm{Ct}$ (cycle threshold) and all the analyzes were performed through $2^{-\Delta \Delta C T}$ method.

For gene expression relative quantification of $I D A$-like and $H A E$-like genes in different plant tissues and organs in wild type plants, every gene expression was normalized respect to NbenPP2A expression. In apical buds, nodes, internodes, corolla, style and stigma, and root tissues, gene expression values were relative to the lowest expression value of each gene in the relevant tissue or organ, within primer sets. In leaf, anthers, fruits and corolla base tissues, gene expression values were relative to the expression value of the earliest developmental stage relevant for that tissue (young leaf, anthers and fruits in stage 2, and corolla base of a stage 1 flower, respectively) within primer sets. Units were represented as the $\log 2$ of the fold change. In the case of leaf, anthers, fruits and base of the corolla tissues, red color indicates that the gene is upregulated (values over 0); white, that remain unchanges (values close to 0 ); and blue, that the gene is downregulated (values under 0); all respect to NbenPP2A expression in the corresponding tissue in its earliest developmental stage.

In the water stress experiment, $N b e n P P 2 A$ was also used as a housekeeping gene for normalization, and watered tissue expression values as a relative reference, thus constituting our control conditions. Conditions were appropriate for each measurement, using the corresponding control tissue (leaf or root of watered plants) as a relative reference. Units were represented as fold change. Red color indicates that the gene is upregulated (values over 1); white, that remain unchanged (values close to 1); and blue, that the gene is downregulated (values under 1); all respect to $N b e n P P 2 A$ expression in the corresponding watered (control) tissue.

\section{Supplementary information}

Supplementary information accompanies this paper at https://doi.org/10 1186/s12870-020-2250-8.

Additional file 1: Nicotiana IDA1 promoters and coding sequences alignment.pdf. Alignment of the $5^{\prime}$-UTR sequences (500 bp) and the CDS of NbenIDA1A, NbenIDA1B, NtabIDA1A, NtabIDA1B, NsyIIDA1 and NtomIDA1 genes. Start codon is highlighted in green, and cis-acting regulatory elements are highlighted as follows: brown line, abscisic acid; blue line, methyl jasmonate; red line, auxins; grey line, drought.

Additional file 2: Solanaceae HSL family.pdf. HAE-like gene families in species of the Solanaceae family, (genome localization from different Sol Genomics Network databases [50])

Additional file 3: HAE-like peptides phylogenetic tree.pdf. Circular phylogenetic tree of HAE-like peptides of Arabidopsis thaliana and several species of the Solanaceae family ( $N$. benthamiana, N. tabacum, $N$. sylvestris, N. tomentosiformis, S. lycopersicum, S. tuberosum, S. melongena and C. annuum).

Additional file 4: Alignment IDA-like prepropeptides.pdf. Sequence alignment of IDA-like peptides from several species of the Solanaceae family ( $N$. benthamiana, N. tabacum, N. sylvestris, N. tomentosiformis, S. lycopersicum, S. tuberosum, S. melongena and C. annuum) and from Arabidopsis. The CINEMA color scheme is used to inform about the chemical nature of the amino acid residues in the EPIP domain (blue, polar positive; red, negative; green, neutral; white, non-polar aliphatic; purple, ocher and yellow, aromatic residues).

Additional file 5: Gene expression raw and final processed data.xlsx. Additional file 6: $\mathrm{qPCR}$ primers.pdf. Primers used for quantitative PCR analysis.

Additional file 7: Solanaceae IDA-like and HAE-like gene families.xlsx. The DNA and protein sequences, as well as the localization of the sequences in the genomes in SolGenomics and the sequence IDs are contained in this file.

\section{Abbreviations}

ABA: Abscisic acid; AUX: Auxins; GA: Gibberellin; HAE: HAESA: IDA: INFLORESCENCE DEFICIENT IN ABSCISSION

\section{Acknowledgements}

We want to acknowledge Dr. Karelia Velázquez and Àngel Boix for preparing and taking care of the $N$. benthamiana plants.

\section{Authors' contributions}

DV, CD, MT and FRT designed the research, DV, CD, and FRT performed the research and analyzed the data, DV, DGI, MT and FRT drafted and substantively revised the article. All authors have read and approved the final manuscript, and ensure that this is the case.

\section{Funding}

Financial support was provided by the Instituto Nacional de Investigación y Tecnología Agraria y Alimentaria, INIA, and Agencia Estatal de Innovación (Ministerio de Ciencia, Innovación y Universidades, Spain) through grants \# RTA-00071-C06-01 and \# RTI2018-097790-R-100, respectively, and the Instituto Valenciano de Investigaciones Agrarias (IVIA), through grant \# 51915.

Funds were used for the design of the study and collection, analysis, and interpretation of data and in writing the manuscript, as well as in the open access payment.

\section{Availability of data and materials}

All data generated or analysed during this study are included in this published article and its supplementary information files. 


\section{Ethics approval and consent to participate}

Not applicable.

\section{Consent for publication}

Not applicable.

\section{Competing interests}

The authors declare that they have no competing interests.

Received: 30 July 2019 Accepted: 14 January 2020

Published online: 20 January 2020

\section{References}

1. Butenko MA, Patterson SE, Grini PE, Stenvik G, Amundsen SS, Mandal A, et al. INFLORESCENCE DEFICIENT IN ABSCISSION controls floral organ abscission in Arabidopsis and identifies a novel family of putative ligands in plants. Plant Cell. 2003;15:2296-307.

2. Patharkar OR, Walker JC. Core mechanisms regulating developmentally timed and environmentally triggered abscission. Plant Physiol. 2016;172:510-20.

3. Shi C, Alling RM, Hammerstad M, Aalen RB. Control of organ abscission and other cell separation processes by evolutionary conserved peptide signaling. Plants. 2019;8:225.

4. Estornell LH, Agustí J, Merelo P, Talón M, Tadeo FR, et al. Plant Sci. 2013; 199-200:48-60.

5. Patharkar OR, Walker JC. Connections between abscission, dehiscence, pathogen defense, drought tolerance, and senescence. Plant Sci. 2019;284:25-9.

6. Patharkar OR, Walker JC. Advances in abscission signaling. J Exp Bot. 2018; 69:733-40.

7. Liu B, Butenko MA, Shi C-L, Bolivar JL, Winge P, Stenvik G-E, et al. NEVERSHED and INFLORESCENCE DEFICIENT IN ABSCISSION are differentially required for cell expansion and cell separation during floral organ abscission in Arabidopsis thaliana. J Exp Bot. 2013:64:5345-57.

8. Niederhuth CE, Patharkar OR, Walker JC. Transcriptional profiling of the Arabidopsis abscission mutant hae hsl2 by RNA-Seq. BMC Genomics. 2013;14:37.

9. Stenvik G-E, Tandstad NM, Guo Y, Shi C-L, Kristiansen W, Holmgren A, et al. The EPIP peptide of INFLORESCENCE DEFICIENT IN ABSCISSION is sufficient to induce abscission in Arabidopsis through the receptor-like kinases HAESA and HAESA-LIKE2. Plant Cell. 2008;20:1805-17.

10. Kumpf RP, Shi C-L, Larrieu A, Stø IM, Butenko MA, Péret B, et al. Floral organ abscission peptide IDA and its HAE/HSL2 receptors control cell separation during lateral root emergence. Proc Natl Acad Sci. 2013;110:5235-40.

11. Shi C-L, von Wangenheim D, Herrmann U, Wildhagen M, Kulik I, Kopf A, et al. The dynamics of root cap sloughing in Arabidopsis is regulated by peptide signalling. Nat Plants. 2018;4:596-604.

12. Vie AK, Najafi J, Liu B, Winge P, Butenko MA, Hornslien KS, et al. The IDA IDA-LIKE and PIP/PIP-LIKE gene families in Arabidopsis: phylogenetic relationship, expression patterns, and transcriptional effect of the PIPL3 peptide. J Exp Bot. 2015;66:5351-65.

13. Vie AK, Najafi J, Winge $P$, Cattan $E$, Wrzaczek $M$, Kangasjärvi J, et al. The IDALIKE peptides IDL6 and IDL7 are negative modulators of stress responses in Arabidopsis thaliana. J Exp Bot. 2017;68:3557-71.

14. Liu C, Zhang C, Fan M, Ma W, Chen M, Cai F, et al. GmIDL2a and GmIDL4a, encoding the Inflorescence Deficient in abscission-like protein, Are Involved in Soybean Cell Wall Degradation during Lateral Root Emergence. Int J Mol Sci. 2018;19:2262.

15. Tucker ML, Yang R. IDA-like gene expression in soybean and tomato leaf abscission and requirement for a diffusible stelar abscission signal. AoB Plants. 2012:2012:pls035.

16. Estornell LH, Wildhagen M, Pérez-Amador MA, Talón M, Tadeo FR, Butenko MA. The IDA Peptide Controls Abscission in Arabidopsis and Citrus. Front Plant Sci. 2015;6:1-7.

17. Stø IM, RJS O, Fooyontphanich K, Jin X, JMB K, Fischer U, et al. Conservation of the abscission signaling peptide IDA during Angiosperm evolution: withstanding genome duplications and gain and loss of the receptors HAE/ HSL2. Front Plant Sci. 2015:6:931.

18. Kim J, Yang J, Yang R, Sicher RC, Chang C, Tucker ML. Transcriptome Analysis of Soybean Leaf Abscission Identifies Transcriptional Regulators of Organ Polarity and Cell Fate. Front Plant Sci. 2016;7:1-16.

19. Wilmowicz E, Kućko A Ostrowski M, Panek K. INFLORESCENCE DEFICIENT IN ABSCISSION-like is an abscission-associated and phytohormone-regulated gene in flower separation of Lupinus luteus. Plant Growth Regul. 2018;85: 91-100.

20. Ying P, Li C, Liu X, Xia R, Zhao M, Li J. Identification and molecular characterization of an IDA-like gene from litchi, LCIDL1, whose ectopic expression promotes floral organ abscission in Arabidopsis. Sci Rep. 2016;6: 37135.

21. Tranbarger TJ, Domonhédo H, Cazemajor M, Dubreuil C, Fischer U, Morcillo F. The PIP peptide of INFLORESCENCE DEFICIENT IN ABSCISSION enhances Populus leaf and Elaeis guineensis fruit abscission. Plants. 2019;8:143.

22. Goodin MM, Zaitlin D, Naidu RA, Lommel SA. Nicotiana benthamiana : its history and future as a model for plant-pathogen interactions. Mol PlantMicrobe Interact. 2008;21:1015-26.

23. Bally J, Jung H, Mortimer C, Naim F, Philips JG, Hellens R, et al. The rise and rise of Nicotiana benthamiana: a Plant for all Reasons. Annu Rev Phytopathol. 2018:56:405-26.

24. Glover NM, Redestig H, Dessimoz C. Homoeologs: what are they and how do we infer them? Trends Plant Sci. 2016;21:609-21.

25. Wu X-M, Yu Y, Han L-B, Li C-L, Wang H-Y, Zhong N-Q, et al. The tobacco BLADE-ON-PETIOLE2 gene mediates differentiation of the Corolla abscission zone by controlling longitudinal cell expansion. Plant Physiol. 2012;159:835-50.

26. Giorio P, Guida G, Mistretta C, Sellami MH, Oliva M, Punzo P, et al. Physiological, biochemical and molecular responses to water stress and rehydration in Mediterranean adapted tomato landraces. Plant Biol. 2018;20: 995-1004.

27. Tani E, Kizis D, Markellou E, Papadakis I, Tsamadia D, Leventis G, et al. Cultivar-Dependent Responses of Eggplant (Solanum melongena L.) to Simultaneous Verticillium dahliae Infection and Drought. Front Plant Sci. 2018:9:1181.

28. Wang Z, Xu W, Kang J, Li M, Huang J, Ke Q, et al. Overexpression of alfalfa Orange gene in tobacco enhances carotenoid accumulation and tolerance to multiple abiotic stresses. Plant Physiol Biochem. 2018;130:613-22.

29. López-Serrano L, Canet-Sanchis G, Vuletin Selak G, Penella C, San Bautista A, López-Galarza S, et al. Pepper Rootstock and Scion Physiological Responses Under Drought Stress. Front Plant Sci. 2019;10:38.

30. Tomato Genome Consortium T. The tomato genome sequence provides insights into fleshy fruit evolution. Nature. 2012;485:635-41.

31. Emanuelsson $O$, Nielsen $H$, Brunak S, von Heijne G. Predicting subcellular localization of proteins based on their $\mathrm{N}$-terminal amino acid sequence. $J$ Mol Biol. 2000;300:1005-16.

32. Almagro Armenteros JJ, Tsirigos KD, Sønderby CK, Petersen TN, Winther $\mathrm{O}$, Brunak S, et al. SignalP 5.0 improves signal peptide predictions using deep neural networks. Nat Biotechnol. 2019;37:420-3.

33. Bailey CD, Koch MA, Mayer M, Mummenhoff K, O'Kane SL Jr, Warwick SI, et al. Toward a global phylogeny of the Brassicaceae. Mol Biol Evol. 2006;23: 2142-60.

34. Stenvik G-E, Butenko MA, Urbanowicz BR, Rose JKC, Aalen RB. Overexpression of INFLORESCENCE DEFICIENT IN ABSCISSION activates cell separation in vestigial abscission zones in Arabidopsis. Plant Cell. 2006;18:1467-76.

35. Aalen RB, Wildhagen M, Stø IM, Butenko MA. IDA: a peptide ligand regulating cell separation processes in Arabidopsis. J Exp Bot. 2013;64:5253-61.

36. Schardon K, Hohl M, Graff L, Pfannstiel J, Schulze W, Stintzi A, et al. Precursor processing for plant peptide hormone maturation by subtilisinlike serine proteinases. Science. 2016;354:1594-7.

37. Clarkson JJ, Knapp S, Garcia VF, Olmstead RG, Leitch AR, Chase MW Phylogenetic relationships in Nicotiana (Solanaceae) inferred from multiple plastid DNA regions. Mol Phylogenet Evol. 2004;33:75-90.

38. Leitch IJ, Hanson L, Lim KY, Kovarik A, Chase MW, Clarkson JJ, et al. The ups and downs of genome size evolution in Polyploid species of Nicotiana (Solanaceae). Ann Bot. 2008;101:805-14.

39. Skalická K, Lim KY, Matyasek R, Matzke M, Leitch AR, Kovarik A. Preferential elimination of repeated DNA sequences from the paternal, Nicotiana tomentosiformis genome donor of a synthetic, allotetraploid tobacco. New Phytol. 2005;166:291-303.

40. Renny-Byfield S, Chester M, Kovařík A, Le Comber SC, Grandbastien M-A Deloger $M$, et al. Next generation sequencing reveals genome downsizing in Allotetraploid Nicotiana tabacum, predominantly through the elimination of paternally derived repetitive DNAs. Mol Biol Evol. 2011;28:2843-54.

41. Renny-Byfield S, Kovařík A, Chester M, Nichols RA, Macas J, Novák P, et al. Independent, Rapid and Targeted Loss of Highly Repetitive DNA in Natural and Synthetic Allopolyploids of Nicotiana tabacum. PLoS One. 2012;7: e36963. 
42. Butenko MA, Stenvik G-E, Alm V, Saether B, Patterson SE, Aalen RB. Ethylenedependent and -independent pathways controlling floral abscission are revealed to converge using promoter:reporter gene constructs in the ida abscission mutant. J Exp Bot. 2006;57:3627-37.

43. Wang G-Q, Wei P-C, Tan F, Yu M, Zhang X-Y, Chen Q-J, et al. The Transcription Factor AtDOF4.7 Is Involved in Ethylene- and IDA-Mediated Organ Abscission in Arabidopsis. Front Plant Sci. 2016;7:863.

44. Basu MM, González-Carranza ZH, Azam-Ali S, Tang S, Shahid AA, Roberts JA. The manipulation of Auxin in the abscission zone cells of Arabidopsis flowers reveals that Indoleacetic acid signaling is a prerequisite for organ shedding. Plant Physiol. 2013;162:96-106.

45. Marciniak K, Kućko A, Wilmowicz E, Świdziński M, Przedniczek K, Kopcewicz $J$. Gibberellic acid affects the functioning of the flower abscission zone in Lupinus luteus via cooperation with the ethylene precursor independently of abscisic acid. J Plant Physiol. 2018;229:170-4.

46. Ben-Cheikh W, Perez-Botella J, Tadeo FR, Talon M, Primo-Millo E. Pollination increases gibberellin levels in developing ovaries of seeded varieties of Citrus. Plant Physiol. 1997;114:557-64.

47. Mehouachi J, Iglesias DJ, Tadeo FR, Agustí M, Primo-Millo E, Talon M. The role of leaves in citrus fruitlet abscission: effects on endogenous gibberellin levels and carbohydrate content. J Hortic Sci Biotechnol. 2000;75:79-85.

48. Rizza A, Jones AM. The makings of a gradient: spatiotemporal distribution of gibberellins in plant development. Curr Opin Plant Biol. 2019;47:9-15.

49. Force A, Lynch M, Pickett FB, Amores A, Yan Y, Postlethwait J. Preservation of duplicate genes by complementary, Degenerative Mutations. Genetics. 1999;151:1531-45.

50. Mueller LA, Solow TH, Taylor N, Skwarecki B, Buels R, Binns J, et al. The SOL genomics network. A comparative resource for Solanaceae biology and beyond. Plant Physiol. 2005;138:1310-7.

51. Bombarely A, Rosli HG, Vrebalov J, Moffett P, Mueller LA, Martin GB. A draft genome sequence of Nicotiana benthamiana to enhance molecular plantmicrobe biology research. Mol Plant-Microbe Interact. 2012;25:1523-30.

52. Edwards KD, Fernandez-Pozo N, Drake-Stowe K, Humphry M, Evans AD, Bombarely $A$, et al. A reference genome for Nicotiana tabacum enables map-based cloning of homeologous loci implicated in nitrogen utilization efficiency. BMC Genomics. 2017;18:448.

53. Sierro N, Battey JN, Ouadi S, Bovet L, Goepfert S, Bakaher N, et al. Reference genomes and transcriptomes of Nicotiana sylvestris and Nicotiana tomentosiformis. Genome Biol. 2013;14:R60.

54. Sierro N, Battey JND, Ouadi S, Bakaher N, Bovet L, Willig A, et al. The tobacco genome sequence and its comparison with those of tomato and potato. Nat Commun. 2014;5:3833.

55. Hulse-Kemp AM, Maheshwari S, Stoffel K, Hill TA, Jaffe D, Williams SR, et al. Reference quality assembly of the 3.5-Gb genome of Capsicum annuum from a single linked-read library. Hortic Res. 2018;5:4.

56. Kumar S, Stecher G, Tamura K. MEGA7: molecular evolutionary genetics analysis version 7.0 for bigger datasets. Mol Biol Evol. 2016;33:1870-4.

57. Saitou N, Nei M. The neighbor-joining method: a new method for reconstructing phylogenetic trees. Mol Biol Evol. 1987;4:406-25.

58. Troadec JP, Gervois A, Oger L. Statistics of Voronoi cells of slightly perturbed facecentered cubic and hexagonal close-packed lattices. Europhys Lett. 1998;42:167-72.

59. Lescot M. PlantCARE, a database of plant cis-acting regulatory elements and a portal to tools for in silico analysis of promoter sequences. Nucleic Acids Res. 2002:30:325-7.

60. Liu W, Xie Y, Ma J, Luo X, Nie P, Zuo Z, et al. IBS: an illustrator for the presentation and visualization of biological sequences: fig. 1. Bioinformatics. 2015;31:3359-61.

61. Untergasser A, Cutcutache I, Koressaar T, Ye J, Faircloth BC, Remm M, et al. Primer3-new capabilities and interfaces. Nucleic Acids Res. 2012;40:e115.

62. Bustin S, Huggett J. GPCR primer design revisited. Biomol Detect Quantif. 2017;14:19-28.

63. Terol J, Nueda MJ, Ventimilla D, Tadeo F, Talon M. Transcriptomic analysis of Citrus Clementina mandarin fruits maturation reveals a MADS-box transcription factor that might be involved in the regulation of earliness. BMC Plant Biol. 2019;19:47.

64. Liu D, Shi L, Han C, Yu J, Li D, Zhang Y. Validation of reference genes for gene expression studies in virus-infected Nicotiana benthamiana using quantitative real-time PCR. PLoS One. 2012;7:e46451.

\section{Publisher's Note}

Springer Nature remains neutral with regard to jurisdictional claims in published maps and institutional affiliations.

\section{Ready to submit your research? Choose BMC and benefit from}

- fast, convenient online submission

- thorough peer review by experienced researchers in your field

- rapid publication on acceptance

- support for research data, including large and complex data types

- gold Open Access which fosters wider collaboration and increased citations

- maximum visibility for your research: over $100 \mathrm{M}$ website views per year

At BMC, research is always in progress.

Learn more biomedcentral.com/submissions 